\title{
LA ESCRITURA HISTÓRICA SOBRE LA INDEPENDENCIA DE CENTROAMÉRICA: VALORACIÓN RECIENTE DE UNA TRAYECTORIA (2010-2020)*
}

\section{THE HISTORICAL WRITING OF THE CENTRAL AMERICAN INDEPENDENCE: AN ASSESSMENT OF THE LAST DECADE (2010-2020)}

\author{
José Cal \\ Universidad de San Carlos de Guatemala
}

\begin{abstract}
SUMARIO: I. EL RETORNO DE LA HISTORIA POLÍTICA.- II. LA INDEPENDENCIA CENTROAMERICANA COMO PROBLEMA HISTORIOGRÁFICO.- III. EL CAMINO RECORRIDO.-
\end{abstract}

Resumen: El estudio propone un recorrido por las transformaciones experimentadas por la escritura histórica sobre la independencia centroamericana a partir de la segunda mitad del siglo XX poniendo especial atención en su desarrollo reciente, dentro del que ha alcanzado un alto grado de especialización para construir un modelo explicativo complejo con una narración más rica y multidimensional de las transformaciones políticas del istmo centroamericano hacia la construcción de prácticas y culturas políticas modernas en las primeras décadas de su historia republicana.

\begin{abstract}
The study suggests a journey through experimented transformations among historical writings regarding Central American independence from the second half of the XX Century; giving special attention to its recent development where it is shown the high level of specialization that it has reached to build a complex explanatory model with a rich and multidimensional narrative of political transformations of the Central American isthmus towards the construction of modern political cultures and practices in the first decades of its republican history.
\end{abstract}

Palabras clave: Independencia centroamericana, Historiografia centroamericana, Historia política, Culturas políticas.

Key Words: Central American Independence, Central American Historiography, Political History, Political Cultures.

\footnotetext{
* Conferencia impartida en la Academia de Geografia e Historia de Guatemala el 7 de septiembre de 2016 y en el Seminario de Metodología de la Historia e Historiografia del Centro de Investigaciones y Estudios en Antropologia Social (CIESAS Peninsular, Mérida, México) el 22 de octubre de 2018.
} 


\section{LA VUELTA DE LA HISTORIA POLÍTICA.}

Centroamérica siempre ha tenido una dimensión relevante dentro de la Historia humana debido a su biodiversidad, los numerosos pueblos que habitan su territorio y a su situación geográfica de pasaje interocéanico que ocupa hasta hoy. No obstante, su inclusión dentro de la Historia universal siempre se constituye en un reto, ya que su presencia en el tiempo es compleja y diversa como su propio territorio y a pesar de guardar rasgos comunes con la Historia latinoamericana la aplicación de este patrón de análisis podría conducirnos a un desconocimiento de su particularmente convulsa historia política en la edad moderna. ${ }^{1}$

Como bien apunta Héctor Pérez Brignoli, todo el istmo fue explorado por las grandes potencias imperiales por razones comerciales, navales y militares, por lo que Centroamérica fue asumida como región bajo la óptica de estos intereses. También en los comienzos del dominio ibérico se construyó una visión regional del territorio centroamericano, considerado en este momento como colonia de segundo orden y su organización administrativa y estatal dio lugar desde finales del siglo XVI a unidades politicas mayores que se constituyeron en núcleos administrativos razonablemente sólidos y pasaron por diversos ensayos administrativos como las gobernaciones e intendencias, dando lugar a la formación de estructuras nacionales de Guatemala hasta Panamá. Esta organización no siempre coincidió con la diversidad territorial y cultural de la región, por lo que hay que reconocer que este proceso de construcción de antiguas provincias a naciones fue débil e incompleto. Por ello, con su emancipación política e intentos posteriores de modernización durante el siglo XIX, la región centroamericana, pese a tener una Historia y rasgos culturales comunes, terminó por fragmentarse. La movilización de recursos económicos y políticos de sus élites propiciaron algunos momentos de integración como el proyecto federal de 1823 y la guerra contra los filibusteros que invadieron Nicaragua en 1856, pero la región reunió en su territorio a pequeños estados débilmente construidos. De manera que la unidad centroamericana no se puede considerar que está dada, sino que ha sido precariamente conservada. No es imposible, pero tampoco debe verse como inevitable o predeterminada por la Historia o los rasgos geográficos. ${ }^{2}$

El último siglo de gobierno español en América Central pasó por un periodo de gran inestabilidad política y depresión económica. Aunque se intentó impulsar un crecimiento y liberalización de la economía mediante las reformas administrativas de mediados del siglo XVIII las pérdidas económicas fueron en aumento por el contrabando. El terremoto que destruyó la capital del Reino en 1773 planteaba un costoso traslado hacia su nuevo asentamiento, tarea en la que el gobierno colonial puso toda su atención y recursos descuidando al resto de las provincias, con lo que aumentó el sentimiento separatista presente dentro del proceso de emancipación política del istmo. La plaga de langosta acabó con las

1 Peter N. Stearns, Una nueva Historia para un mundo global. Introducción a la $<<$ World History >>, Crítica, Barcelona, 2012, p. 105.

\footnotetext{
2 Héctor Pérez Brignoli, “Transformaciones del espacio centroamericano”, en Marcelo Carmagnani, Alicia Hernández Chávez y Ruggiero Romano (coords.), Para una historia de América II. Los nudos (1), El Colegio de México - Fondo de Cultura Económica (FCE), México, 1999, pp. 55-93.
} 
cosechas entre 1799 y 1805 y la producción textil entró en declive debido al aumento de las importaciones extranjeras. Esta caída de las actividades económicas redujo los ingresos de la administración colonial, quedando sin recursos para pagar las obras públicas y los salarios de su burocracia, situación que se hizo más dramática cuando los comerciantes de la capital realizaron un donativo patriótico de un millón de pesos para auxiliar a las tropas españolas en su resistencia a la presión napoléonica en 1808. Junto a estas penurias, las noticias sobre los movimientos independentistas en México y levantamientos dentro de la propia América Central eran más frecuentes. La inestabilidad política de España y su incapacidad para defender su comercio y una acción exterior respetable sumadas al colapso de la economía guatemalteca condujo a las élites centroamericanas a aceptar la independencia como alternativa y a suscribirla cuando se constituyó en oportunidad. Este periodo profundizó la fragmentación entre las denominadas Provincias Unidas de la América del Centro (1823) y condujo a una lucha interna y guerras civiles que se prolongaron por lo menos medio siglo más. Ante estos hechos, Ralph Lee Woodward subraya que Centroamérica inició su periplo independiente bajo un desarrollo caótico e insatisfactorio conforme a facciones radicales y moderadas de las élites que emergían para luchar entre sí y fueron haciéndola más dependiente de la agroexportación, modelo económico que generó un crecimiento excluyente y comunidades políticas con derechos ciudadanos restringidos. El primer medio siglo de independencia centroamericana transcurrió entre las luchas entre las facciones políticas de las élites por impulsar o retrasar el crecimiento económico y la modernización política ${ }^{3}$ en la que su adhesión intelectual a esta modernidad no tuvo necesariamente una inmediata transferencia social, por lo que la tensión entre esta aspiración de cambio y la prevalencia de comportamientos propios de una sociedad del Antiguo Régimen moldearon la cultura politica de las sociedades centroamericanas. ${ }^{4}$

La peculiaridad y complejidad de la emancipación politica del istmo ha sido un foco de atención sobre el que se ha desarrollado la consolidación y alcance interpretativo de la historiografia centroamericana en las últimas décadas. Al situarla en el complejo proceso de transición hacia la modernidad desde una perspectiva regional y de larga duración para comprenderla en toda su amplitud transformadora de las relaciones políticas empezó a gestarse una revalorización la Historia politica para comprender el pasado regional. ${ }^{5}$ La renovación experimentada por las ciencias humanas durante el siglo XX al poner su atención en los procesos de construcción de subjetividad puso especial atención en

\footnotetext{
3 Ralph Lee Woodward Jr., Rafael Carrera y la creación de la República de Guatemala, 18211871, Centro de Investigaciones Regionales de Mesoamérica (CIRMA) - Plumsock Mesoamerican Studies (PMS), Vermont, 2002, pp. 2-23.

4 Michel Bertrand, "Élites sociales de Guatemala en vísperas de la Independencia", en Izaskun Álvarez Cuartero y Julio Sánchez Gómez (eds.), Visiones y revisiones de la independencia americana. México, Centroamérica y Haití, Ediciones Universidad de Salamanca, Salamanca, 2005, p. 59.

5 José Cal, “'Un largo y penoso proceso': Severo Martínez Peláez y la Historia social sobre la independencia de Centroamérica", en Coralia Gutiérrez Álvarez (coord.), La primavera en la memoria. Historia y política en Severo Martínez Peláez, Benemérita Universidad Autónoma de Puebla, Puebla, 2020, p. 157.
} 
comprender lo politico como una dimensión fundamental de la vida cotidiana de los sujetos y no solamente relacionada con la esfera estatal. ${ }^{6}$ Este cambio metodológico se desarrolló en Centroamérica desde los años noventa cuando los historiadores Arturo Taracena y Jean Piel dieron impulso a la Historia regional con la finalidad inicial de comprender aspectos desconocidos de los procesos de formación de los nacientes estados nacionales centroamericanos en el siglo XIX situando el análisis desde las provincias y regiones y ya no desde las capitales. Esta perspectiva dio lugar a una reescritura de la Historia política en el istmo, la que puso en evidencia que el estado colonial estaba conformado por regiones con problemáticas, intereses, fronteras y culturas particulares que incidieron en la construcción de estados nacionales bajo una lógica administrativa autoritaria y centralista, la que contribuyó a su consolidación o desestructuración. ${ }^{8}$ Bajo estas nuevas perspectivas, como bien señala Xiomara Avendaño, la escritura histórica en Centroamérica se dirigió a la comprensión del complejo fenómeno político que se dio entre los años 1821 y $1823 .{ }^{9}$ El rompimiento del vínculo de América Central con la monarquía hispánica para construir un Estado moderno se desarrolló bajo un entrelazamiento problemático entre ideas antiguas y modernas que también estuvo presente en la formación de estructuras estatales, las que emergieron sobre la base del municipio castellano sin existencia de partidos políticos y donde los pobladores expresaban sus ideas mediante el cabildo, ámbito en el que se empezaron a redefinir las pertenencias a la comunidad política de los distintos sectores sociales: criollos, mestizos y comunidades indígenas. ${ }^{10}$ Asimismo, la independencia fue la consecución de las aspiraciones de las élites centroamericanas de tomar decisiones administrativas por su cuenta para avanzar económicamente y contar una representación política plena que les permitiese formar un gobierno federativo. Su proclamación es considerada, bajo su óptica, como la cristalización del deseo de las mayorías, pero en la que deben tomar la dirección del proceso para canalizarla

6 Como bien señala Guillermo Palacios, la Nueva Historia Politica integró componentes del mundo de la cultura y de las prácticas sociales muchas veces distantes (u opuestos) del Estado como nunca se habian presentado en los estudios históricos. Guillermo Palacios, Introducción, en Guillermo Palacios (coord.), Ensayos sobre la Nueva Historia Politica de América Latina, Siglo XIX, El Colegio de México, México, 2007, p. 13.

7 José Cal, "Las identidades politicas y étnicas en la Historia regional de Guatemala: reflexiones sobre un recorrido", Anuario de Historia Regional y de las Fronteras, n ${ }^{\circ} 1,2009$, pp. 159-172.

8 Arturo Taracena Arriola, "Reflexiones sobre la región histórica. Los Altos de Guatemala y Yucatán en la primera mitad del siglo XIX", Bulletin de l'Institut Français d'Études Andines, n ${ }^{\circ}$, 2018, pp. 41-55.

9 Xiomara Avendaño, Centroamérica entre lo antiguo y lo moderno: institucionalidad, ciudadanía y representación politica, 1810-1838, Publicaciones de la Universitat Jaume I, Castellón, 2009 , p. 39.

10 Xiomara Avendaño, "La independencia de Guatemala y El Salvador: una nueva visión sobre los actores", en Manuel Chust y José Antonio Serrano (coords.), Debates sobre las independencias iberoamericanas, Asociación de Historiadores Latinoamericanistas Europeos (AHILA) Iberoamericana Vervuert, 2007, pp. 237-254. Xiomara Avendaño, "El gobierno provincial en el Reino de Guatemala, 1821-1823", en Virginia Guedea (coord.), La independencia de México y el proceso autonomista novohispano, 1808-1824, Universidad Nacional Autónoma de México (UNAM) - Instituto de Investigaciones Dr. José María Luis Mora, 2001, p. 329. 
institucionalmente y evitar la acción de los sectores populares. Su proclamación no podría considerarse como un momento histórico de integración política del istmo, ya que fue recibida de manera diferenciada al ser vista no solamente como una separación de España sino también de Guatemala y ser considerada como una situación de orfandad política, la que llevaría inevitablemente a su anexión al naciente imperio mexicano. ${ }^{11}$

La escritura histórica en Centroamérica ha conseguido, como ha sucedido dentro del impulso de la Nueva Historia Politica en América Latina, ubicar la complejidad y peculiaridad de la emancipación política del istmo dentro de procesos más amplios en espacio y tiempo con el que ha extendido su alcance interpretativo. ${ }^{12}$ Más allá de asumir un lustre de novedad metodológica, ha mostrado una preocupación por indagar en las acciones políticas de los diferentes sujetos y grupos de interés que nacen y se desarrollan en el espacio político, dando consecución al alejamiento de la Historia fáctica de las biografias políticas y de las conmemoraciones con las que actualmente los gobiernos centroamericanos vuelven al relato de la independencia como gesta cívica, dejando de lado toda esa Historia escrita por historiadores centroamericanos y centroamericanistas que nos era desconocida hasta hace pocos años y que podría llevar a una reflexión nodal en el espacio público acerca de la construcción de la democracia y la ciudadanía en la región. ${ }^{13}$ Dada la centralidad de lo político en la Historia del istmo es dificil pensar que la historiografia centroamericana ha efectuado un retour a la Historia política. No obstante, consideramos que su propio avance no solamente condujo a cuestionar y arrumbar en buena medida la escritura de una Historia política centrada en élites, hechos y gobernada por el sectarismo, sino ante todo, a mostrar que la Historia politica es lo suficientemente amplia para que tengan cabida en ella procedimientos de análisis más complejos y una mayor riqueza documental, ya que no debe eludirse que la escritura de la historia siempre desempeña un papel político en la sociedad, independientemente de que fuese o no su objetivo. ${ }^{14} \mathrm{El}$ discurso histórico, al pasar por una apropiación social, aporta conocimientos, argumentos e interpretaciones al discurso político. Por esta razón, pensar la política centroamericana siempre requerirá de una mirada hacia la Historia para poder describirla y explicarla criticamente en los alcances y debilidades de las acciones de sus ciudadanos y la formación de sus instituciones. Hablar de una presencia renovada de la Historia política dentro de la escritura histórica en Centroamérica pasa por una comprensión compleja del poder como un ámbito de relaciones, de tensiones y de construcción de procesos, actores, sujetos y lenguajes politicos, en los que la independencia dejó de ser una mera fecha o narración para transformarse en una pregunta a responder que generó un programa de investigación que sigue contribuyendo a la madurez de la

11 David Díaz Arias, “Independencia”, en Jordana Dym y Sajid Herrera (coords.), Centroamérica durante las revoluciones atlánticas, vocabulario político, 1750-1850, IIESFORD Editores, San Salvador, 2014, p. 133.

12 José Cal, “'Un largo y penoso proceso': Severo Martínez Peláez y la Historia social sobre la independencia de Centroamérica”, op. cit., p. 158.

13 Marcelo Carmagnani, "Campos, prácticas y adquisiciones de la Historia Política Latinoamericana”, en Guillermo Palacios, op. cit., p. 31.

14 Elena Hernández Sandoica, Tendencias historiográficas actuales. Escribir Historia hoy, Ediciones Akal, Madrid, 2004, p. 425. 
historiografia centroamericana a pesar de las acusadas debilidades institucionales de la región para dedicarse profesionalmente a la investigación histórica.

\section{LA INDEPENDENCIA CENTROAMERICANA COMO PROBLEMA HISTORIOGRÁFICO.}

La independencia empezó a ser repensada dentro de la vida intelectual del istmo desde inicios del siglo $\mathrm{XX}, 15$ periodo en el que predominaron las historias nacionales ${ }^{16}$ hasta que en 1964 el sociólogo salvadoreño Alejandro Dagoberto Marroquín en su obra Apreciación sociológica de la independencia salvadoreña ${ }^{17}$ cuestionó el relato histórico oficial que la exaltaba como gesta cívica y suscitó gran polémica en los círculos intelectuales y políticos de El Salvador al abordarla bajo una visión de proceso desde la lucha de clases y los intereses económicos de la élite provincial sansalvadoreña. ${ }^{18}$ Pocos años después, en 1971, el historiador guatemalteco Severo Martínez en La Patria del Criollo habló de la proclamación de una independencia conservadora que no había transformado la situación de los sectores subalternos y que debía dejar de ser vista como un tema cívico para transformarse en un problema científico. ${ }^{19}$ En Costa Rica, Rodolfo Cerdas Cruz también había propuesto en 1967 una interpretación de los años iniciales de la independencia del país más lejano del centro político del istmo desde la perspectiva de la lucha de clases y la influencia que ejerció en la consolidación de

15 El lingüista salvadoreño Rafael Lara-Martínez, en su libro El Bicentenario: un enfoque alternativo expone cómo la sociedad letrada El Ateneo, fundada en San Salvador en 1912, realiza proposiciones críticas en el espacio público sobre la escasa trascendencia de la independencia centroamericana. En Guatemala, el abogado y diplomático Virgilio Rodríguez Beteta, argumenta en su obra Ideologías de la independencia, publicada en 1926, que la independencia tuvo una proyección política fracasada debido a que no consiguió la unidad política de la región y predominó en su proclamación un contenido aristocrático que le impidió salir del antiguo régimen y tener un sistema político democrático. Rafael Lara-Martínez, El Bicentenario: un enfoque alternativo, Editorial Universidad Don Bosco, San Salvador, 2011, pp. 59-96. José Cal, "La indagatoria del pasado de Virgilio Rodríguez Beteta (1885-1967): un acercamiento a su contribución a la historiografia guatemalteca y centroamericana", Revista Expedições, n 1, 2017, pp. 1-15.

16 Xiomara Avendaño y Guillermo Fernández Ampié, "Actores políticos e instituciones en la Intendencia de Nicaragua y Costa Rica: una relectura de la aceptación del acta emancipadora (1821) y la unión a México (1822)", en Sajid Herrera y Elizet Payne (coords.), La justa libertad de los pueblos. Las independencias en Centroamérica, Chiapas y Panamá, Sílex Ediciones, Madrid, 2021 (en prensa).

17 Alejandro Dagoberto Marroquín, Apreciación sociológica de la independencia salvadoreña, Dirección de Publicaciones e Impresos (DPI), San Salvador, 2000.

18 José Cal, “'Un largo y penoso proceso': Severo Martínez Peláez y la Historia social sobre la independencia de Centroamérica", op. cit., p. 154. Xiomara Avendaño, "Alejandro Dagoberto Marroquín y su 'Apreciación sociológica de la independencia salvadoreña", Cuadernos de Ciencias Sociales, $\mathrm{n}^{\circ}$ 1, 2011, pp. 16-26. Carlos Gregorio López Bernal, "Historiografia y movimientos sociales en El Salvador (1811-1932): un balance preliminar", Revista de Historia, $\mathrm{n}^{\circ}$ 67, 2013, p. 96.

19 Severo Martínez Peláez, Centroamérica en los años de la independencia, el país y los habitantes, Departamento de Publicaciones, Facultad de Ciencias Económicas - Universidad de San Carlos de Guatemala, Guatemala, 1977, p. 3 
la élite de la ciudad de San José en la vida política del país. ${ }^{20}$ Julio César Pinto Soria habia orientado su trayectoria de investigación al preparar su doctorado en la Universidad de Leipzig (1978) a la formación de los estados nacionales centroamericanos desde una perspectiva marxista, pero incursionando en una escritura histórica del proceso nutrida de diversidad de fuentes de archivo, atenta a la dinámica social, a sus implicaciones económicas y a su dimensión regional. Fue en esta misma década que Severo Martínez se había planteado la escritura de un libro dedicado integramente al análisis de la independencia centroamericana con material del Archivo General de Indias y aunque este libro nunca se escribió se puede apreciar que en estos años la independencia centroamericana ya ocupaba un lugar central en las preocupaciones historiográficas de los historiadores centroamericanos y dedicados a Centroamérica. Aunque la presencia del marxismo tradicional dentro de la escritura histórica en el istmo impulsó una rigidez analítica y conceptual que hacía imposible una visión histórica con pretensión totalizante de una región tan diversa y compleja, ${ }^{21}$ fue un punto de partida fundamental para que la independencia fuese planteada como una pregunta a responder y se transformara en los años posteriores en un programa de investigación, situación que la llevó a salir no solamente de la esfera conmemorativa estatal y los relatos históricos nacionales, sino que también se redimensionara como un problema historiográfico que debía ser abordado desde distintas dimensiones y actores. ${ }^{22}$

Las élites también dejaron de predominar en la escritura histórica posterior. Los historiadores Chester Zelaya en Nicaragua, Rafael Obregón Loría en Costa Rica y Héctor Humberto Samayoa Guevara y Jorge Luján Muñoz en Guatemala empezaron a escribir trabajos que no utilizaron las biografias politicas como punto de partida de la narrativa histórica de los hechos e incluir la presencia de otros sectores sociales en el proceso de independencia y comprender su recepción diferenciada en toda la región. El abogado e intelectual peruano Andrés Towsend Ezcurra prosiguió esta ruta al analizar la breve existencia de la república centroamericana como un proceso que se originó en la propia emancipación politica para dar lugar a su desintegración. El peso de los intereses comerciales de las élites de la capital de Guatemala y de las provincias, los intentos de construcción estatal desde la fiscalidad y un cada vez más creciente abordaje de la historia del pensamiento político con especial atención al constitucionalismo gaditano se hicieron presentes en los trabajos de historiadores estadounidenses como Ralph Lee Woodward Jr., Troy S. Floyd, Miles L. Wortman y Mario Rodriguez en la década de los ochenta. En estos mismos años, el abogado guatemalteco Jorge Mario García Laguardia desde la historia constitucional planteó la necesidad de situar el proceso en la dimensión atlántica del

20 Iván Molina, "La influencia del marxismo en la historiografia costarricense", A contra corriente, $\mathrm{n}^{\circ}$ 2, 2008, p. 226.

21 Xiomara Avendaño, "La independencia de Guatemala y El Salvador: una nueva visión sobre los actores”, op. cit., pp. 237-254.

22 José Cal, “'Un largo y penoso proceso': Severo Martínez Peláez y la Historia social sobre la independencia de Centroamérica”, op. cit., p. 154. 
pensamiento político iberoamericano y dar una mirada de región al proceso de implantación del constitucionalismo moderno. ${ }^{23}$

Al haberse abierto estos campos de trabajo historiográfico, Timothy Ana y José Antonio Fernández mostraron sus estudios la necesidad de seguir analizando la independencia centroamericana desde una mirada regional y fueron las investigaciones de Adolfo Bonilla, Jordana Dym, Sajid Herrera, Xiomara Avendaño y Mario Vázquez las que abrieron nuevas perspectivas para repensarla desde los ámbitos de la construcción del pensamiento político, la representación, las elecciones y la participación ciudadana. ${ }^{24}$ Los trabajos de François Xavier Guerra habian influido decisivamente en el estudio de las independencias iberoamericanas y permitieron revelar la peculiaridad de la situación centroamericana, caracterizada por la ruptura de vínculos con la metrópoli a causa de la depresión económica, la presión exterior de los acontecimientos de México y el control político del proceso ejercido por la élite criolla que impidió su construcción como entidad politica regional haciendo más compleja la viabilidad de sus estados nacionales, situación que terminó por replicar los mismos resultados de conflicto e inestabilidad institucional que tuvieron lugar en todos los países latinoamericanos. También en este momento, la investigación histórica sobre las independencias iberoamericanas empezaba a dirigirse al estudio de las culturas politicas: una herramienta metodológica especialmente versátil y fecunda para abordar fenómenos, experiencias, lenguajes $\mathrm{y}$ trayectorias politicas desde una perspectiva transnacional, con la que la independencia fue vista dentro de procesos de ruptura más amplios y su lugar como hecho politico vinculado a sistemas de representaciones compartidas o rechazadas por amplios y diversos grupos en el seno de una sociedad y el lugar que ocupan en ella desde su relación con el poder. Esta perspectiva permite visibilizar y hacer inteligibles comportamientos que sin tenerla en cuenta podrian parecer inexplicables. Desde este punto de partida, se ha ido realizando una revisión de la historiografia tradicional sobre el periodo, pero ante todo, se han explorado nuevas dimensiones que estaban presentes en su propia complejidad, en las que el historiador busca comprender estos sistemas de representaciones relacionados con marcos de referencia colectivos, compartidos o temporales, los que están formados por instituciones, normas, prácticas, valores, discursos e ideologías que muestran cómo los hechos y el comportamiento públicos están condicionados contextualmente. ${ }^{25}$

Esta transformación en la historiografia centroamericana sobre la independencia se puede apreciar, siguiendo a Coralia Gutiérrez, ${ }^{26}$ en que las antiguas historias nacionales pasaron a ser de carácter local y regional para

${ }^{23}$ Ibid., p. 155.

${ }^{24}$ Ibid., p. 156.

25 Nuria Tabanera y Marta Bonaudo, Introducción, en Nuria Tabanera y Marta Bonaudo (coords.), América Latina. De la independencia a la crisis del liberalismo, 1810-1930 (Historia de las culturas políticas en España y América Latina. Vol. V), Marcial Pons Historia - Prensas de la Universidad de Zaragoza, Madrid, 2014, p. 10

26 Coralia Gutiérrez Álvarez, "La historiografia contemporánea sobre la independencia en Centroamérica", Nuevo Mundo. Mundos Nuevos, 2009, https://doi.org/10.4000/nuevomundo.54642. 
entender otras dimensiones del proceso de emancipación política centroamericana desde una perspectiva de larga duración, ${ }^{27}$ en la que se aprecian las transformaciones de las ideas de comunidad política y soberanía municipal por las que las antiguas provincias se convirtieron en estados y donde el constitucionalismo gaditano impulsa la redefinición de las pertenencias a la comunidad política de los criollos, los mestizos y las comunidades indigenas. Un fenómeno político de amplio espectro en el que con la formación de instituciones en el gobierno provincial los criollos consolidaron un proceso de autonomía que llevaba muchas décadas de gestación y que numerosas poblaciones indígenas reivindicaron para preservar la integridad de sus autoridades tradicionales. Después de la emancipación, los indígenas y mestizos encontrarán en el municipio y el ayuntamiento importantes espacios de articulación administrativa y política para asumir algunas funciones del Estado. ${ }^{28}$ La historiografia centroamericana ha mostrado que la propia fractura del sistema monárquico llevó a las élites del istmo a buscar un nuevo horizonte político en la anexión al imperio mexicano, la que, al no tener un anclaje social sólido, se desvaneció en los dificultosos caminos de reafirmación nacional de México y los estados centroamericanos. La trayectoria trazada por la escritura histórica centroamericana sobre la independencia considerada más allá del hecho, en sus antecedentes y repercusiones, ha dado también a conocer cómo las élites de las distintas regiones aprendieron a movilizar recursos para defender sus derechos y prerrogativas, tanto del que consideraban un imperio lejano como el de sus actores más inmediatos y a pesar de que buscaron impulsar la introducción de instituciones representativas en el istmo no promovieron su integración ni una modernidad política en la fundación de sus estados nacionales. Los conocimientos aportados por la historiografia de la independencia centroamericana no solamente redimensionan y esclarecen lo sucedido hace dos siglos en su vida politica, también se constituyen una posibilidad de que la Historia sea el punto de partida para repensar a una Centroamérica que incluya a los sectores sociales que han quedado fuera de algunos de sus logros económicos y políticos y fortalezca el desarrollo de sus instituciones como la de sus pares en otras realidades geográficas. ${ }^{29}$ Hablar de Historia política en Centroamérica es ofrecer una reflexión profunda sobre su paso en el tiempo como una comunidad política diversa pero con un pasado compartido.

Con la llegada del nuevo siglo aparecieron en el horizonte de los pueblos centroamericanos las conmemoraciones bicentenarias al aliento de las celebraciones impulsadas por el gobierno de México. En este momento repensar las independencias hispanoamericanas ya era una tarea de larga trayectoria en la

27 Ya en la década de los setenta, el destacado historiador guatemalteco Jorge Luján Muñoz sostenía que la única forma de estudiar y comprender adecuadamente la independencia era estudiarla como un fenómeno de larga duración. Jorge Luján Muñoz, La independencia y la anexión de Centroamérica a México, Serviprensa Centroamericana, Guatemala, 1982, p. 32.

28 Mónica Quijada, "El pueblo como actor histórico. Algunas reflexiones sobre municipalismo y soberanía en los procesos políticos hispánicos", en Nuria Tabanera y Marta Bonaudo, op. cit., p. 19. Un abordaje más detallado sobre esta temática en: Xiomara Avendaño Rojas, "La institucionalidad jurídico-electoral en Centroamérica durante el siglo XIX: ciudadanía y sufragio", Journal of Iberian and Latin American Research, n² 20, 2014, pp. 19-35.

29 Alcides Hernández, La integración de Centroamérica: desde la Federación a nuestros dias, Departamento Ecuménico de Investigaciones (DEI), San José, 1994. 
historiografia internacional y una preocupación que estaba presente desde hacía mucho tiempo en la escritura histórica centroamericana. Con todo, esta situación también favoreció la profusión de seminarios, congresos y grupos de investigación en torno a la cuestión de las independencias y a numerosas iniciativas de colaboración académica entre instituciones de toda Iberoamérica. ${ }^{30}$ Vino una verdadera avalancha editorial de libros y artículos que vinieron a cubrir algunos vacíos históricos e historiográficos o a ampliar y profundizar caminos ya recorridos sobre cuestiones fundamentales de los procesos de independencia iberoamericanos, con los que se pone de manifiesto la importancia y fecundidad de esta agenda científica en la que sigue vigente la investigación de temas como la participación de la mujer, la diversidad y heterogeneidad de determinados grupos sociales -indigenas, pardos, morenos y esclavos-, el papel del clero, la importancia de la historia regional y el estudio de las culturas politicas en su conjunto entre muchos otros temas. ${ }^{31}$ Las independencias iberoamericanas son consideradas objeto de historia ${ }^{32}$ dentro de la historiografia, pero este proceso ha puesto el reto de analizar una producción que ya podría ser considerada como inabarcable. ${ }^{33}$ El año 2009 marca un ciclo creciente de publicaciones sobre las independencias americanas que no se centraban únicamente en ofrecer nuevas evidencias sobre su desarrollo, sino plantear revisiones del camino recorrido por su escritura histórica. Dirigiremos nuestra atención a los trabajos publicados en y sobre Centroamérica en la última década, los que muestran no solamente el importante avance de la investigación histórica registrado en la región en los últimos treinta años, ${ }^{34}$ sino la consolidación de una historiografia especializada en uno de los periodos cruciales de su Historia.

30 Cristóbal Aljovin de Losada, "El Bicentenario y las redes académicas españolas", Argumentos. Revista de análisis y crítica, $\mathrm{n}^{\circ}$ 4, 2010, https://argumentos-historico.iep.org.pe/articulos/elbicentenario-y-las-redes-academicas-espanolas (ISSN 2076-7722).

31 Ivana Frasquet, "Estados y revoluciones en Iberoamérica. A propósito de las independencias en la década de 1820", en Manuel Chust y Rogelio Altez (coord.), Las revoluciones en el largo siglo XIX latinoamericano, AHILA - Iberoamericana Vervuert, 2015, pp. 65-67.

32 El libro compilado por Veronique Hebrárd y Geneviève Verdo reivindica el estudio de las independencias hispanoamericanas desde distintas temporalidades y no solamente desde la larga duración, desde los lenguajes políticos, religiosos y constitucionales, desde la diversidad de sus actores y prácticas y desde sus diversos vínculos con Europa y Estados Unidos. Veronique Hebrárd y Geneviève Verdo, Las independencias iberoamericanas: un objeto de historia, Casa de Velázquez, Madrid, 2013.

33 Debe tenerse en cuenta que solamente Manuel Chust acumula más de un centenar de capítulos de libros y artículos sobre el estudio de las independencias, a lo que deben sumarse las decenas de libros que ha publicado como autor o editor. A inicios del año 2011 el académico del área de Historia de América del Consejo Superior de Investigaciones Científicas de España (CSIC), Antonio Gutiérrez Escudero, recopiló un primer repertorio bibliográfico sobre la historiografia de las independencias hispanoamericanas, el que reunió más de cien títulos y aporta un panorama de referencia para el estudioso sobre el desarrollo de este campo de trabajo historiográfico. Antonio Gutiérrez Escudero, "La emancipación hispanoamericana y los bicentenarios: un primer repertorio bibliográfico", Araucaria, n 25, 2011, pp. 207-227.

34 Murdo Mac Leod, "Nuevas perspectivas sobre la Historia colonial de Centroamérica entre 1520 y $1720 ”$, Mesoamérica, n 50, 2008, pp. 159-191. 


\section{EL CAMINO RECORRIDO.}

Centroamérica ha sido un tema de investigación con una presencia tenue e intermitente dentro del americanismo español y europeo, tradicionalmente centrados en los territorios con mayor peso económico y político de América Latina, como son los casos de México y Perú, situación que se hizo extensiva a la profusa producción historiográfica centrada en reflexionar sobre las independencias hispanoamericanas. ${ }^{35}$ A pesar de ello, desde el año 2007 se puede apreciar que Centroamérica empieza a aparecer en la historiografia especializada sobre la independencia que se está impulsando dentro del medio académico de habla española desde diversas iniciativas científicas. En el libro de Manuel Chust y José Antonio Serrano publicado en el año 2007 por la Asociación de Historiadores Latinoamericanistas Europeos (AHILA) titulado Debates sobre las independencias iberoamericanas aparece un sugerente estudio de la historiadora nicaragüense Xiomara Avendaño en el que revisa la historiografia del proceso de independencia de Guatemala y El Salvador con atención a los trabajos de Alejandro Dagoberto Marroquín y Julio César Pinto Soria. ${ }^{36}$ Y dentro de esta misma colección, el volumen dirigido por Ivana Frasquet y Andrea Slemian dedicado al estudio de los procesos de formación de estados nacionales en América Latina desde la independencia, se incluye un trabajo de la historiadora estadounidense Jordana Dym dedicado a reflexionar, desde la construcción histórica del espacio, sobre el proceso de formación del estado de Guatemala como consecuencia del efecto desintegrador de la emancipación política centroamericana, sin dejar de resaltar su peculiaridad respecto a lo sucedido en el resto de América Latina. ${ }^{37}$ La incansable actividad académica de Manuel Chust al frente del grupo de investigación Procesos revolucionarios liberales en España y México. De la independencia al estado nación, ha dado como resultado la formación de redes académicas de alcance latinoamericano y mundial dentro de las que han participado los historiadores centroamericanos más destacados en el estudio de esta temática, Xiomara Avendaño y Sajid Herrera, quienes publicaron sus tesis doctorales como libros en los años 2009 y 201438 dentro del programa de publicaciones dedicados a la Historia de América en la Universitat Jaume I, 39 las que son consideradas fundamentales para entender la complejidad de la evolución de la institucionalidad y las prácticas politicas, antiguas y modernas que formaron parte del proceso de formación del estado y el sistema republicano en el istmo. La publicación en el año 2009 del libro de los historiadores españoles

35 W. George Lovell, Conquest and survival in Colonial Guatemala: a historical Geography of the Cuchumatan highlands, 1500-1821, McGill - Queen's University Press, Montreal, 1992, p. xviii.

36 Xiomara Avendaño, "La independencia de Guatemala y El Salvador: una nueva visión sobre los actores", op. cit., pp. 237-254.

37 Jordana Dym, "La república de Guatemala: la emergencia de un país, 1808-1851" en Ivana Frasquet y Adrea Slemian (eds.), De las independencias iberoamericanas a los estados nacionales (1810-1850). 200 años de Historia, AHILA - Iberoamericana Vervuert, 2007, pp. 217-242.

38 Xiomara Avendaño, Centroamérica entre lo antiguo y lo moderno: institucionalidad, ciudadanía y representación política, 1810-1838, Publicaciones de la Universitat Jaume I, Castellón, 2009.

39 Sajid Herrera, El ejercicio de gobernar. Del cabildo borbónico al ayuntamiento liberal, El Salvador colonial 1750-1821, Publicaciones de la Universitat Jaume I, Castellón, 2014. 
Manuel Chust e Ivana Frasquet Las independencias en América, ${ }^{40}$ ya dedicaba un apartado específico a la independencia centroamericana, la que hasta hacía pocos años era solamente una breve mención en muchos de los proyectos historiográficos de la década precedente.

En el libro Jaque a la corona. La cuestión politica en las Independencias Iberoamericanas, dirigido por el historiador mexicano Juan Ortiz Escamilla e Ivana Frasquet encontramos tres contribuciones de historiadores centroamericanos: un análisis historiográfico de la independencia salvadoreña por Xiomara Avendaño, un análisis de las prácticas electorales en la provincia de El Salvador del historiador salvadoreño Sajid Herrera y uno de los trabajos de revisión historiográfica más completos sobre la independencia de Centroamérica escrito por la historiadora guatemalteca Coralia Gutiérrez Álvarez, especialista en el periodo colonial.41 La escritora peruana Sara Beatriz Guardia ha hecho numerosos esfuerzos editoriales por mostrar la presencia de la mujer dentro de las independencias y su libro: Las mujeres en la independencia de América Latina publicado en el 2010, incluye una colaboración de la lingüista guatemalteca Guillermina Herrera sobre la escritora decimonónica Lola Montenegro. ${ }^{42}$ Herrera publicó en el año 2012 un volumen compilatorio sobre las mujeres en el bicentenario, en el que se reúnen colaboraciones en torno a la participación de distintas mujeres intelectuales en la creación de la República de Guatemala. ${ }^{43}$ Nuevamente hay presencia de historiadores centroamericanos, Xiomara Avendaño y Sajid Herrera, en un libro editado por Manuel Chust en el año 2010 con el que rompió la lógica habitual de las publicaciones de este periodo al reunir a un grupo de reconocidos especialistas en las independencias hispanoamericanas para que respondan a preguntas de gran profundidad sobre el proceso, iniciativa con la que se mostró claramente su carácter multidimensional y complejo trazando un estado de la cuestión bastante esclarecedor sobre el avance de las investigaciones sobre el tema en Iberoamérica. ${ }^{44} \mathrm{El}$ estudio del constitucionalismo gaditano abarca otro volumen de producción sobre el que es dificil hacer una valoración de conjunto, pero hay que resaltar durante esta década la publicación dirigida por el historiador Alberto Ramos Santana dirigida a esclarecer la huella de la constitución de Cádiz en

\footnotetext{
40 Manuel Chust e Ivana Frasquet, Las independencias en América, Los Libros de la Catarata, Madrid, 2009.

41 Sajid Herrera, "La práctica electoral en la provincia/estado de El Salvador, 1821-1839", Xiomara Avendaño Rojas, "El proceso de independencia en El Salvador: análisis historiográfico de los movimientos precursores”, Coralia Gutiérrez Álvarez, "La historiografia contemporánea sobre la independencia en Centroamérica", en Juan Ortiz Escamilla (coord.), Jaque a la corona. La cuestión política en las independencias iberoamericanas, Publicaciones de la Universitat Jaume I, Castellón, 2010, pp. 251-272. 291-341.

42 Guillermina Herrera, "Lola Montenegro. La romántica centroamericana", en Sara Beatriz Guardia (edit.), Las mujeres en la independencia de América Latina, Centro de Estudios de la Mujer en la Historia de América Latina (CEMHAL), Lima, 2010, pp. 455-468.

43 Guillermina Herrera Peña, Mujeres en el bicentenario. Aportes femeninos en la creación de la República de Guatemala, Organización de las Naciones Unidas para la Educación, la Ciencia y la Cultura (UNESCO), Guatemala, 2012.

44 Manuel Chust (edit.), Las independencias iberoamericanas en su laberinto. Controversias, cuestiones, interpretaciones, Publicaciones de la Universidad de Valencia, Valencia, 2010.
} 
América, estudio en el que se incluyeron las colaboraciones de los historiadores Rodrigo Quesada Monge (Costa Rica), Arturo y Luis Pedro Taracena Arriola (Guatemala), Yesenia Martínez García (Honduras), Alfredo Castillero Calvo (Panamá) 45 y nuevamente de Sajid Herrera (El Salvador) y Xiomara Avendaño (Nicaragua). ${ }^{46}$ La Embajada de España en Honduras impulsó en el año 2012 una publicación específica sobre el bicentenario de la constitución gaditana, en la que participaron Miguel Cálix Suazo, Yesenia Martínez, Rolando Sierra y Edgar Soriano, ${ }^{47}$ historiador que un año después publicó el trabajo: 1812: la rebeldía popular en Tegucigalpa en el contexto de los levantamientos en Centroamérica (1809-1812),48 investigación que basada en la historiografia existente y documentación local de archivo intenta mostrar los movimientos políticos de rechazo a la corona en esta provincia del antiguo Reino de Guatemala. Esta temática, con mayor diversidad y presencia de fuentes de archivo, es estudiada por el historiador hondureño Pedro Quiel en su tesis de Maestria en Historia. ${ }^{49}$ En el libro editado por Ivana Frasquet: Jamás ha llovido reyes el cielo. De independencias, revoluciones y liberalismos en Iberoamérica, Sajid Herrera aporta un esclarecedor estudio sobre el constitucionalismo liberal español en la historiografia centroamericana, ${ }^{50}$ con el que establece una ruta de comprensión de los idearios politicos que nutrieron la historiografia liberal, de gran influjo en la escritura histórica del istmo en los siglos XIX y XX. Jordana Dym registra una

45 El autor, con motivo de las conmemoraciones sobre el bicentenario, publicó en 2012 un artículo de revisión de sus propias investigaciones sobre la independencia de Panamá de España: Alfredo Castillero Calvo, "Independencia de Panamá de España. Para el Bicentenario: nuevas evidencias y reflexiones", Tareas, n 141, 2012, pp. 101-128.

46 Rodrigo Quesada Monge, “Costa Rica y la Constitución de Cádiz de 1812”, Sajid Alfredo Herrera Mena, "La herencia del liberalismo hispánico en Centroamérica. Libertad de imprenta, prensa y espacio público moderno en El Salvador, 1810-1890", Arturo Taracena Arriola y Luis Pedro Taracena Arriola, "Guatemala en las Cortes de Cádiz", Yesenia Martínez, "La influencia de la Constitución de Cádiz en la concepción de la libertad en Honduras", Xiomara Avendaño, "La influencia gaditana en Nicaragua: las elecciones indirectas durante el sistema monárquico constitucional, 1811-1823", Alfredo Castillero Calvo, "Las Cortes de Cádiz y la independencia de Panamá”, en Alberto Ramos Santana, La constitución de Cádiz y su huella en América, Servicio de Publicaciones de la Universidad de Cádiz, Cádiz, 2011, pp. 169-174.187-210.219-228.

47 Miguel Cálix Suazo, “¿Influyó la Constitución de Cádiz en la independencia de Centroamérica?", Yesenia Martínez, "La influencia de la Constitución de Cádiz en la concepción de la libertad en Honduras", Rolando Sierra Fonseca, "Los objetivos estratégicos de la provincia de Honduras en las Cortes de Cádiz", Edgar Soriano, "Una aproximación a las experiencias del juramento de la Constitución de Cádiz en el contexto de Tegucigalpa (1812-1820)", en Varios autores, Bicentenario de la constitución de Cádiz en Honduras, Agencia Española de Cooperación Internacional para el Desarrollo (AECID), Tegucigalpa, 2012, pp. 17-60.73-106.

48 Edgar Soriano, 1812: la rebeldia popular de Tegucigalpa en el contexto de los levantamientos en Centroamérica (1809-1812), Ñ Editores, Tegucigalpa, 2013.

49 Pedro Antonio Quiel Morales, "Mueran todos los chapetones y que vivan los criollos": la Alcaldía Mayor de Tegucigalpa entre reforma y crisis, 1762-1817, Tesis Maestría en Historia, Centro de Investigaciones y Estudios Superiores en Antropología Social (CIESAS Peninsular), 2018, 228 pp.

50 Sajid Herrera, "El constitucionalismo liberal español en la historiografia centroamericana (siglo XIX): elogios, silencios y descalificaciones", en Ivana Frasquet, (edit.), Jamás ha llovido reyes el cielo. De independencias, revoluciones y liberalismos en Iberoamérica, Quito, Corporación Editora Nacional - Universidad Andina Simón Bolivar, 2013. 
trayectoria de investigación en la que ha puesto especial dedicación al estudio de la independencia centroamericana. Siguiendo el modelo explicativo global del historiador británico David Armitage ${ }^{51}$ se ha centrado en el estudio de las actas de independencia y del constitucionalismo gaditano en la región, temática sobre la que publicó un estudio ampliamente documentado en el libro dirigido por el historiador mexicano Roberto Breña titulado: Cádiz a debate: actualidad, contexto y legado, publicado por El Colegio de México en el año 2014.52 Dym participó en la dirección de un libro colectivo junto a los historiadores mexicanos Erika Pani y Alfredo Ávila dedicado al estudio de los textos fundamentales sobre las independencias americanas en una coedición entre El Colegio de México y la Universidad Nacional Autónoma de México (UNAM), dentro del que aporta un estudio que propone una comprensión histórica evolutiva sobre el proceso de independencia, poniendo atención al proceso de suscripción de las actas para explicar ampliamente los cambios institucionales registrados en el periodo. ${ }^{53}$ Otra publicación de interés es la que dirigió el historiador argentino Carlos Malamud en 2012 dedicada a reflexionar sobre el reconocimiento dado por España a las independencias latinoamericanas, la que se incluyó en la colección América Latina en la Historia Contemporánea de la Fundación MAPFRE, libro que incluye las colaboraciones de la historiadora Ligia Madrigal Mendieta sobre Nicaragua, del abogado Jorge Francisco Sáenz Carbonell para el caso de Costa Rica, de los historiadores José Cal, Carlos Moreno y Olga Joya en los estudios sobre Guatemala, El Salvador y Honduras y del abogado Arístides Royo, quien escribió el artículo sobre Panamá. ${ }^{54}$ Dentro de esta misma colección se publicó en 2013 el libro: Tiempos de revolución. Comprender las independencias iberoamericanas, de Manuel Chust e Ivana Frasquet en el que muestran la madurez de su itinerario de investigación al presentar una visión de conjunto amplia y documentada sobre los procesos de independencia hispanoamericanos como uno de los acontecimientos más relevantes de la Historia universal contemporánea y en el que Centroamérica ocupa nuevamente un lugar específico. ${ }^{55}$ El Instituto Nacional de Estudios Históricos de las Revoluciones de México (INEHRM) publicó en 2010 un libro sobre las independencias iberoamericanas, en el que se incluye una contribución de Sajid Herrera sobre los levantamientos y protestas acaecidos en El Salvador en el año 1811, hecho al que se le ha dado gran presencia en las narrativas históricas sobre el lugar de El Salvador en la independencia centroamericana desde la esfera gubernamental y

51 David Armitage, Las declaraciones de independencia. Una historia global, Marcial Pons, Madrid, 2012.

52 Jordana Dym, “Cádiz en Centroamérica, 1808-1826. Una historia documental”, en Roberto Breña, Cádiz a debate: actualidad, contexto y legado, El Colegio de México, México, 2014, pp. 113-123.

53 Jordana Dym, "Declarar la independencia: la evolución de la independencia centroamericana, 1821-1864", en Alfredo Ávila, Jordana Dym y Erika Pani, Las declaraciones de independencia. Los textos fundamentales de las independencias americanas, El Colegio de México - UNAM, México, 2013, pp. 217-242.

54 Carlos Malamud Rickles, Ruptura y reconciliación. España y el reconocimiento de las independencias latinoamericanas, Fundación MAPFRE - Taurus, Madrid, 2012, pp. 125-168.239268.331-352.

55 Manuel Chust e Ivana Frasquet, Tiempos de revolución. Comprender las independencias iberoamericanas, Fundación MAPFRE - Taurus, Madrid, 2013. 
que ha sido ampliamente debatido por el propio Herrera y la historiadora salvadoreña Eugenia López Velásquez en diversos artículos especializados. ${ }^{56}$ El trabajo de Herrera también fue publicado en un número especial de la revista de la Universidad de El Salvador dedicado a los levantamientos de 1811, en el que se incluyen otras contribuciones de académicos y funcionarios gubernamentales. ${ }^{57}$ La historiadora mexicana Patricia Galeana publicó otro libro de Historia latinoamericana centrado en los procesos de independencia, en los que se incluyen colaboraciones de Jorge Mario García Laguardia en torno a los conceptos de monarquía constitucional y república en la historia centroamericana y del historiador mexicano Mario Vázquez Olivera sobre la influencia del Plan de Iguala en el proceso independentista salvadoreño. 58

Javier Fernández Sebastián impulsó otro programa de investigación de gran alcance sobre como el de Manuel Chust. Desde el grupo de investigación Historia Intelectual de la Politica Moderna (Lenguajes, conceptos y discursos) impulsó redes de colaboración académica para el desarrollo de numerosas publicaciones. Una de ellas referida a los primeros liberalismos en el mundo iberoamericano, compilación en la que colaboró con un artículo sobre Centroamérica el historiador costarricense Víctor Hugo Acuña Ortega, en el que retoma una idea ya desarrollada por el historiador salvadoreño Adolfo Bonilla ${ }^{59}$ sobre la presencia de diversas corrientes del liberalismo dentro de la vida politica del istmo en los años previos a la independencia. $60 \mathrm{Su}$ proyecto historiográfico de mayor aliento ha sido el Diccionario politico y social del mundo iberoamericano, dividido en dos partes. En la segunda, que consta de 10 volúmenes, hay una importante participación de historiadores centroamericanos y centroamericanistas con destacadas

56 Sajid Herrera, “1811. Relectura de los levantamientos y protestas en la provincia de San Salvador", en Loudes Martínez Ocampo, Las independencias iberoamericanas, Comisión Organizadora de las Conmemoraciones de 2010, México, 2012, pp. 119-138. Sajid Herrera, "Versiones y usos de la Historia desde el Estado salvadoreño: a propósito del bicentenario (18112011)", Revista de Historia, n 70, 2014, pp. 143-158. Eugenia López Velásquez, "Los motines de 1811 contra el mal gobierno en la intendencia de San Salvador. Una perspectiva distinta a la del $<<$ primer grito de independencia>>", en Varios autores, Miradas para pensar el siglo XX. E1 Salvador 1880-1990: Historiografia contemporánea, Ministerio de Educación, San Salvador, 2018, pp. 55-92.

57 La Universidad. San Salvador 1811, n 16, 2011, 194 pp.

58 Mario Vázquez Olivera, "El Plan de Iguala y la independencia de San Salvador”, Jorge Mario García Laguardia, "La independencia de la Capitanía General de Guatemala. El dilema del nuevo régimen: Monarquía constitucional o República”, en Patricia Galeana (coord.), Historia comparada de las Américas. Sus procesos independentistas, Siglo XXI Editores, México, 2010, pp. 399-467.

59 Adolfo Bonilla, The Central American enlightenment 1770-1838: an interpretation of political ideas and political history, Tesis Doctor en Filosofia, University of Manchester, 1996, 491 pp. En 2013, Bonilla publicó un artículo en el que hace un nuevo recorrido por las ideas y argumentos de su disertación doctoral, proponiendo que Centroamérica, bajo una dirección política sabia y prudente, tuvo posibilidades históricas reales de llegar a ser una república moderna exitosa. Adolfo Bonilla, "Establecer una república en un contexto inadecuado es como ensillar una vaca: la discusión política en torno a la independencia de Centroamérica", Revista de Humanidades y Ciencias Sociales, $\mathrm{n}^{\circ} 5,2013$, pp. 15-36.

60 Víctor Hugo Acuña Ortega, "El liberalismo en Centroamérica en tiempos de la independencia (1810-1850)", en Javier Fernández Sebastián (coord.), La aurora de la libertad. Los primeros liberalismos en el mundo iberoamericano, Marcial Pons, Madrid, 2012, pp. 117-144. 
trayectorias de investigación. Junto a Sajid Herrera, Xiomara Avendaño y Jordana Dym colaboraron en los diversos volúmenes los historiadores costarricenses David Díaz Arias y José Antonio Fernández, el historiador hondureño Rolando Sierra Fonseca, la historiadora española Sonia Alda Mejías, el historiador francés Christophe Belaubre y el historiador estadounidense Douglass Sullivan-González. ${ }^{61}$ El historiador guatemalteco Óscar Peláez Almengor colabora en otro libro editado en España al albor de las conmemoraciones sobre la independencia bajo la coordinación del escritor Manuel Gaete, en el que aporta un trabajo que estudia la influencia militar española en la formación del ejército nacional guatemalteco que establece sus antecedentes en el periodo colonial y de emancipación política en 1821.62 Otra publicación que sigue el camino de una reflexión conmemorativa es el libro coordinado por el filósofo español Jacinto Choza: La independencia de América. Primer centenario $y$ segundo centenario publicada en el 2011,63 en el que los artículos dedicados a Centroamérica estuvieron a cargo del economista español Guillermo Vázquez Vicente. Su propósito principal es comparar las conmemoraciones del primer y segundo centenario de la independencia de América, tema sobre el que hay contribuciones sobre países como Argentina, Chile y Venezuela y gran parte con enfoque latinoamericano. Entre los años 2019 y 2020 Manuel Chust publica dos libros que exploran nuevas perspectivas y temáticas relacionadas con las independencias hispanoamericanas: Los miedos sin patria: temores revolucionarios en las independencias iberoamericanas en colaboración con la historiadora peruana Claudia Rosas Lauro, ${ }^{64}$ libro en el que participan con una contribución sobre Centroamérica los historiadores guatemaltecos Arturo Taracena Arriola y Juan Carlos Sarazúa Pérez, quienes abordan cómo las élites intelectuales y politicas centroamericanas, temerosas de una situación de revolución que trastocara su posición preponderante, realizan una apropiación del término en sus escritos y actuaciones públicas para legitimar la posición de su facción política. Taracena y Sarazúa forman parte del importante desarrollo alcanzado por la Historia política en la región. En el

61 Los conceptos trabajados para este diccionario por los historiadores centroamericanos y centroamericanistas son: Civilización (Sajid Herrera), Democracia (Jordana Dym), Estado (Sajid Herrera y Xiomara Avendaño), Independencia (David Díaz), Libertad (Rolando Sierra), Orden (Jordana Dym), Partido (Sonia Alda Mejias y Christophe Belaubre), Patria (Douglass SullivanGonzález), Revolución (José Antonio Fernández) y Soberanía (Jordana Dym). Javier Fernández Sebastián (dir.), Diccionario politico y social del mundo iberoamericano. Conceptos fundamentales, 1770-1870 (Iberconceptos II. 10 vols.), Universidad del País Vasco - Centro de Estudios Politicos y Constitucionales, Madrid, 2014.

62 Oscar Peláez Almengor, "Antonio García Pérez y la tradición militar hispana en Guatemala", en Manuel Gaete Jurado (coord.), América y España: un siglo de independencias, Iberdrola, Bilbao, 2014, pp. 293-314.

63 Guillermo Vázquez Vicente, "Nacimiento y ocaso de la Federación de Centroamérica: ¿realidad o juego de intereses?, en Jacinto Choza (coord.), La independencia de América: primer centenario y segundo centenario, Thémata, Sevilla, 2011, pp. 163-187.

64 Arturo Taracena y Juan Carlos Sarazúa, "Centroamérica: del miedo a la apropiación de la 'revolución", en Manuel Chust y Claudia Rosas Lauro (coords), Los miedos sin patria: temores revolucionarios en las independencias iberoamericanas, Sílex Ediciones, Madrid, 2019, pp. 293315. 
segundo libro titulado: ¡Mueran las cadenas! El trienio liberal en América, ${ }^{65}$ Chust da impulso a la perspectiva del trienio liberal para situar y redimensionar contextualmente la proyección política de las independencias hispanoamericanas, el cual incluye un destacado estudio de Mario Vázquez Olivera, en el que recorre la situación centroamericana entre 1820 y 1823 apoyado no solamente por su profundo conocimiento de la historia de la región, sino también por los conocimientos aportados por su ampliamente reconocida investigación sobre la anexión de Centroamérica a México publicada en el año 2009 por el Fondo de Cultura Económica, ${ }^{66}$ la que sigue siendo considerada de referencia para el estudio de la Historia mexicana y centroamericana del siglo XIX. El libro de Vázquez Olivera: Chiapas mexicana. La gestación de la frontera entre México y Guatemala durante la primera mitad del siglo XIX, publicado en $2018,{ }^{67}$ es una aportación fundamental para el estudio de los procesos posteriores al rompimiento de los vínculos de la región con el imperio español, especialmente dentro del proceso de definición de sus fronteras territoriales y políticas. Junto al historiador español Pedro Rújula, Chust publicó en 201968 un libro de síntesis interpretativa sobre el trienio liberal en España que se trata en conjunto con los territorios de la monarquía hispana partiendo de la idea de su pertenencia a una misma entidad politica, intento en el que los autores consiguen una muy bien lograda síntesis de la Historia centroamericana del periodo a partir de sus propias investigaciones y los avances registrados por la historiografia de la región que motivan este escrito. Entre los estudios que analizan procesos políticos que están alrededor de la independencia centroamericana está el libro: Conspiración, guerras y revoluciones politicas en la América hispano-portuguesa, 1808-1824 a cargo de Sajid Herrera.69 Publicado en el 2013 y con poca circulación en la región, reúne estudios sobre los movimientos previos a la emancipación política americana en el Reino de Guatemala, Río de la Plata, Nueva Granada, Nueva España y Brasil. Una publicación que no es habitual para el medio académico centroamericano, pero que reviste de especial importancia para la docencia en el área de Historia Latinoamericana en las aulas universitarias. Bajo la perspectiva de análisis del trienio liberal está el libro de Xiomara Avendaño: El Trienio Constitucional. Moderación, innovación y autonomía en el Reino de Guatemala, 1820-1823,70 en el que reúne varios de sus trabajos ya publicados con la finalidad de mostrar entidades, mecanismos y prácticas políticas en el antiguo

65 Mario Vázquez Olivera, “Centroamérica en la senda de Cádiz, 1820-1823”, en Manuel Chust (edit.), ¡Mueran las cadenas! El trienio liberal en América, Comares, Granada, 2020, pp. 77-114.

66 Mario Vázquez Olivera, El imperio mexicano y el Reino de Guatemala. Proyecto politico y campaña militar, 1821-1823, FCE, México, 2009.

67 Mario Vázquez Olivera, Chiapas mexicana. La gestación de la frontera entre México y Guatemala en la primera mitad del siglo XIX, Centro de Investigaciones en América Latina y el Caribe (CIALC) - Centro de Investigaciones Multidisciplinarias sobre Chiapas y la Frontera Sur (CIMSUR-UNAM), México, 2018.

68 Pedro Rújula y Manuel Chust, El trienio liberal en la monarquía hispánica. Revolución e independencia (1820-1823), Los Libros de la Catarata, Madrid, 2019.

69 Sajid Herrera, Conspiraciones, guerras y revoluciones politicas en la América hispanoportuguesa, 1808-1824, UCA Editores, San Salvador, 2013.

70 Xiomara Avendaño, El trienio constitucional: moderación, innovación y autonomía en el Reino de Guatemala, 1820-1823, UCA Editores, San Salvador, 2018. 
Reino de Guatemala entre 1820 y 1823 hasta su desintegración. Bajo la intencionalidad de proseguir la escritura de una Historia de las culturas politicas está el libro de la historiadora hondureña Ethel García Buchard, quien con su trabajo: Prácticas electorales y cultura politica en Honduras durante el siglo XIX (1812-1894) muestra las dificultades del proceso de construcción de ciudadanía en Honduras al adoptar procedimientos republicanos de sucesión de poder mediante elecciones que eran aplicados en un contexto en el que aún predominaban los mecanismos de expresión politica del antiguo régimen, como eran los levantamientos y pronunciamientos. Investigaciones como las de García Buchard representan la perspectiva adoptada por la historiografia centroamericana de analizar y comprender la independencia dentro de los tensos procesos de transformación política por los que pasó toda la región centroamericana hacia la implantación de una cultura politica moderna. En esta dirección, debe agregarse que el campo de trabajo historiográfico de la Historia fiscal impulsada por los jóvenes historiadores Juan Carlos Sarazúa y Pablo Rodríguez Solano ${ }^{72}$ se inscribe en el amplio ejercicio de contextualización del cambio político en Centroamérica en las primeras décadas del siglo XIX. ${ }^{73}$ Sus contribuciones en la publicación Formación de los estados nacionales centroamericanos $^{74}$ aporta información económica y social del periodo para una valoración más profunda del proceso, lo que también puede decirse de la investigación de la historiadora española Clara Pérez Fabregat sobre el oriente salvadoreño ${ }^{75}$ y la tesis doctoral de la historiadora salvadoreña Eugenia López, ${ }^{76}$ trabajos en los que las transiciones politicas son vistas desde las dimensiones del territorio, las instituciones, las actividades económicas, la fiscalidad, los conflictos entre grupos sociales y la violencia. La inquietud que manifestaba Coralia Gutiérrez sobre una importante presencia en la historiografia centroamericana de estudios dedicados al análisis de la modernidad política en detrimento de una Historia económica $y$ social de mayor alcance ${ }^{77}$ se empieza a

71 Ethel García Buchard, Prácticas electorales y cultura politica en Honduras durante el siglo XIX (1812-1894), Editorial Guaymuras, Tegucigalpa, 2017.

72 Rodríguez Solano publicó una revisión del proceso de independencia costarricense en el que busca esclarecer las bases pactistas de los Estados centroamericanos del periodo. Pablo Augusto Rodríguez Solano, "Derecho Natural en Costa Rica (1821-1823): una revisión crítica del proceso de independencia y formación de los estados en Centroamérica", Iles i Imperis, n ${ }^{\circ} 15,2013$, pp. 55-73.

73 Dos artículos que contribuyen a enriquecer esta perspectiva son: Jordana Dym, "El poder en La Nueva Guatemala: la disputa sobre los alcaldes de Barrio, 1761-1821", Cuadernos de Literatura, $\mathrm{n}^{\circ}$ 28, 2010, pp. 196-229. Aaron Pollack, "Protesta en Patzicía. Los pueblos de indios y la vacatio regis en el reino de Guatemala”, Revista de Indias, n 272, 2018, pp. 147-173.

74 Varios autores, Formación de los estados centroamericanos, s.d.e., San José, 2014.

75 Clara Pérez Fabregat, San Miguel y el oriente salvadoreño. La construcción del Estado de El Salvador, 1780-1865, UCA Editores, San Salvador, 2018.

76 María Eugenia López Mejía Velásquez, Pueblos de indios, de ladinos y de mulatos de San Salvador y Sonsonate en tiempos de reformas y transiciones politicas (1737-1841), Tesis Doctorado en Ciencias Sociales, El Colegio de Michoacán, 2017, 336 pp.

77 Coralia Gutiérrez Álvarez, "La historiografia contemporánea sobre la independencia en Centroamérica", Nuevo Mundo. Mundos Nuevos, 2009, https://doi.org/10.4000/nuevomundo.54642 
satisfacer con la aparición de estos estudios que solamente pueden ser posibles al contar con una historiografia no solamente profusa, sino también diversa en sus escalas de análisis, opciones metodológicas y diversidad de fuentes.

Bajo una perspectiva de conmemoración y de ofrecer una narración histórica sobre lo sucedido en Nicaragua durante la independencia centroamericana, la Academia de Geografia e Historia de Nicaragua publicó este año 2021 dos libros. El primero, con el título: Nicaragua: independencia $y$ frustración republicana (1808-1854), ${ }^{78}$ en el que se reúnen ensayos de los estudiosos de la Historia nicaragüense Jorge Eduardo Arellano y Pablo Kraudy orientados al análisis del proceso de independencia de Nicaragua y el papel de los juristas y otros intelectuales del periodo utilizando principalmente fuentes bibliográficas y secundarias. El segundo, denominado: Perspectivas nicaragüenses de la independencia centroamericana ${ }^{79}$ editado por Ligia Madrigal Mendieta y Jorge Eduardo Arellano, es una recopilación de estudios recientes de algunos miembros de esta corporación académica sobre las repercusiones de la independencia en Nicaragua, de piezas escritas por otros destacados intelectuales del país que la integraron en distintos periodos de su Historia y de una sección de fuentes sobre el tema. Se puede considerar que en ambas obras predomina una perspectiva más tradicional de la Historia política en la que se echa en falta un mayor diálogo con la historiografia centroamericana actual que ayude a superar su perspectiva predominantemente local. La historiadora guatemalteca Ana Maria Urruela de Quezada dirigió la publicación: Memoria y compromiso: La Iglesia y el bicentenario de la Independencia, 1821-2021, con la que se quiere visibilizar la presencia histórica de la Iglesia católica durante de la independencia y que reúne ensayos escritos por presbíteros y profesionales de distintas áreas de conocimiento referidos a temáticas históricas sumamente diversas, por lo que es una publicación que está más dentro de un impulso de conmemoración. Aunque contiene artículos interesantes sobre la Historia de la Iglesia en Guatemala durante el periodo escritos por varios historiadores profesionales guatemaltecos, la contribución del historiador nicaragüense Horacio Cabezas Carcache es la que reviste de mayor interés para esta revisión, al centrarse en la actividad evangelizadora de la Iglesia católica en Guatemala en los años previos a la independencia. ${ }^{80}$ Cabezas Carcache publicó en el 2010 el libro: Independencia centroamericana. Gestión y ocaso del "Plan Pacífico", dirigido a analizar el Plan Pacífico de Independencia promovido por la familia Aycinena para mantener el control del sistema político con apoyo del imperio mexicano, temática desde la que propone una narrativa histórica con perspectiva regional de la independencia de utilidad para los estudiosos, en la que muestra las diferencias entre las élites centroamericanas que dieron lugar a los levantamientos en años previos a la

78 Jorge Eduardo Arellano y Pablo Kraudy Medina, Nicaragua: independencia y frustración republicana, Fondo Editorial El Güegüense - Instituto Nicaragüense de Cultura, Managua, 2021.

79 Jorge Eduardo Arellano y Ligia Madrigal Mendieta (eds.), Perspectivas nicaragüenses de la independencia centroamericana, Academia de Geografia e Historia de Nicaragua, Managua, 2021.

80 Ana María Urruela de Quezada (edit.), Memoria y compromiso: La Iglesia y el bicentenario de la Independencia, 1821-2021 (Tomo I. Tiempos de cambio, 1767-1811), Conferencia Episcopal de Guatemala, Guatemala, (CEG), 2019. 
declaración de 1821.81 Otra contribución de interés sobre la situación de la Iglesia católica durante el periodo es la del historiador estadounidense Robert Holden titulada La Iglesia y la independencia del Reino de Guatemala, en la que propone un recorrido por la posición del clero ante la emancipación y la creciente importancia que cobró la religión como problema político, tema que aborda de manera exhaustiva para el caso de El Salvador la tesis doctoral en Historia eclesiástica de Luis Ernesto Ayala Benitez leída en el 2007 en la Universidad Gregoriana de Roma. ${ }^{82}$

La madurez alcanzada por la historiografia centroamericana queda reflejada en iniciativas de investigación y publicaciones que ponen de manifiesto lo señalado por Murdo Mac Leod al decir que su situación actual se debe en gran medida a que cuenta con generaciones de historiadores e historiadoras cada vez más numerosas, profesionales y eclécticas en sus abordajes y métodos. ${ }^{83}$ Junto a la renovación de la Historia política, su contacto con otras temáticas, corrientes y métodos de la historiografía internacional propiciado por la sociedad de la información y la cooperación académica, ha conseguido elaborar modelos explicativos más complejos sobre el paso del istmo del antiguo régimen hacia la modernidad. Los historiadores costarricenses Ronny Viales y David Diaz impulsaron en el año 2012 la publicación de un libro que fuese una primera reunión de trabajos para reflexionar sobre el bicentenario, la formación de naciones y el desarrollo político de la región. El libro Independencias, Estados y Politica(s) en la Centroamérica del siglo XIX. Las huellas históricas del bicentenario, ${ }^{84}$ publicado en 2012 , reunió estudios divididos en dos secciones. La primera, dedicada a las independencias, contiene un estudio de Jordana Dym centrado nuevamente en el análisis de las actas de independencia y los coordinadores del libro publicaron dos artículos en los que buscaron analizar la independencia desde perspectivas poco abordadas: su impacto en la economia del istmo y los limites de su desarrollo institucional. Estos dos trabajos son dos propuestas metodológicas sobre las que se conducirán sus publicaciones posteriores, en las que la independencia será estudiada desde los efectos

81 Horario Cabezas Carcache, Independencia centroamericana. Gestión y ocaso del "Plan Pacifico", Editorial Universitaria, Guatemala, 2010.

82 Robert H. Holden, "La Iglesia y la independencia del Reino de Guatemala", en Josep Ignasi Saranyana y Juan Bosco Amores Carredano (eds.), Politica y religión en la independencia de la América hispana, Biblioteca de Autores Cristianos (BAC) - Universidad de Navarra, Madrid, 2011, pp. 65-83. Luis Ernesto Ayala Benitez, La iglesia y la independencia política de Centro América: "el caso de el Estado de El Salvador" (1808 - 1833), Tesis Doctorado en Historia Eclesiástica, Universidad Gregoriana, 2007. Otro estudio que está dentro de la misma temática: Manuel Benavides, "El Padre indio Tomás Ruiz, ¿Prócer de la independencia centroamericana?”, Revista de Historia de América, $\mathrm{n}^{\circ}$ 142, 2010, pp. 123-157.

83 Murdo Mac Leod, "Nuevas perspectivas sobre la Historia colonial de Centroamérica entre 1520 y 1720”, Mesoamérica, n 50, 2008, p. 191.

84 Jordana Dym, "Actas de independencia: de la Capitanía General de Guatemala a la República Federal de Centroamérica", David Diaz Arias y Ronny Viales Hurtado, "El impacto económico de la independencia en Centroamérica, 1760-1840. Una interpretación”, David Diaz Arias y Ronny Viales Hurtado, "La felicidad prometida y sus limites. Desarrollo institucional, inclusión/exclusión social y el legado colonial en Centroamérica, 1770-1870", en David Diaz Arias y Ronny Viales Hurtado, Independencias, Estados y Politica(s) en la Centroamérica del siglo XIX. Las huellas históricas del bicentenario, Centro de Investigaciones Históricas de América Central (CIHAC) - Universidad de Costa Rica (UCR), San José, 2012, pp. 3-62. 
económicos desfavorables que provocó en la región y desde las debilidades del proyecto institucional republicano que se empezaba a construir. En la segunda parte se incluyen estudios relacionados con la formación estatal y su dimensión fiscal y politica y otros con mayor atención a las dificultades para establecer una cultura politica moderna en el istmo. El interés de Viales y Díaz Arias por analizar las variables económicas y de inclusión y exclusión social se estructuró como un programa de investigación que dio lugar a la publicación de un libro bajo su coordinación considerado pionero en la historiografia centroamericana actual: Historia de las desigualdades sociales en América Central. Una visión interdisciplinaria, siglos XVII-XXI, en el que se reúnen treinta trabajos de académicos de distintas áreas de conocimiento reunidos en tres secciones: exclusiones económicas, exclusiones sociopolíticas y exclusiones socioculturales. Dentro de la sección de exclusiones sociopolíticas los coordinadores incluyeron el trabajo: 'Sociedad imaginada': el ideario politico de la integración excluyente en Centroamérica, 1821-1870, en el que plantean que aunque el periodo de estudio está lleno de hechos políticos conocidos, se requiere de una historización de las ideas y opciones politicas de unas élites centroamericanas que vieron en la emancipación política la realización del ideario de un primer liberalismo que abria la posibilidad de establecer sociedades más justas e igualitarias, aunque debe indagarse cuánto estuvieron dispuestas a ceder para ir estableciendo este nuevo pacto social. ${ }^{85}$ Aaron Pollack coordinó un libro que puede ser valorado como una de las obras más representativas de la Nueva Historia Politica que se escribe en y sobre la región centroamericana y Chiapas. La época de las independencias en Centroamérica y Chiapas. Procesos politicos y sociales, ${ }^{86}$ es producto del trabajo del grupo de investigación que bajo el liderazgo de Pollack se ha consolidado con sucesivas reuniones académicas en las que han debatido e intercambiado experiencias reconocidos especialistas en Historia del siglo XIX en el istmo. La obra abarca un arco de tiempo que comienza desde la ilustración y llega hasta el debate de anexión al imperio mexicano y recoge la diversidad de perspectivas de análisis alcanzada por la historiografia centroamericana al estudiar las ideas y simbolos políticos, las redes sociales, las rebeliones y la represión. El libro hace una aportación fundamental para situar a Centroamérica en los procesos de independencia hispanoamericanos: no hubo en la región un ejército independentista, un conflicto armado y héroes nacionales vinculados con la independencia. La obra, como indica Pollack, muestra el distanciamiento de la historiografia actual de la independencia en y sobre la región de los análisis predominantemente económicos y sociales y transita también en las rutas de la Historia cultural e intelectual. La introducción de esta obra, escrita por Pollack, puede considerarse junto al trabajo Coralia Gutiérrez otro de los estados de la cuestión más documentados sobre la historiografia de la independencia centroamericana. Jordana Dym y Sajid Herrera, bajo inspiración del proyecto de Iberconceptos dirigido por Fernández Sebastián, publicaron: Centroamérica

85 Ronny Viales y David Díaz Arias, “'Sociedad imaginada': el ideario político de la integración excluyente en Centroamérica, 1821-1870”, en Ronny Viales Hurtado y David Diaz Arias, Historia de las desigualdades sociales en América Central. Una visión interdisciplinaria, Siglos XVIII-XXI, CIHAC - UCR, San José, 2016, pp. 197-218.

86 Aaron Pollack (coord.), La época de las independencias en Centroamérica y Chiapas. Procesos politicos y sociales, Instituto Mora - Universidad Autónoma Metropolitana (UAM), México, 2013. 
durante las revoluciones atlánticas: el vocabulario politico, 1750-1850,87 libro que representa la importancia e influencia que tiene la Historia conceptual dentro de la renovación historiográfica de la región, la que no se puede comprender sin la dedicación que han puesto los historiadores centroamericanos y centroamericanistas al vocabulario sociopolitico como una herramienta metodológica fundamental para comprender las numerosas transformaciones del periodo de emancipación. Puede considerarse como uno de sus principales aportes esclarecer al estudioso que muchos de los conceptos que aparecieron en los lenguajes politicos a inicios del siglo XIX centroamericano no surgieron en la era de las independencias y ya tenian una presencia extendida durante el periodo borbónico. 88 Con motivo del I Simposio internacional de Historia Centroamericana: independencias y formación de Estados Nacionales (1821-1860) celebrado en Costa Rica en 2016, se publicó otro libro de compilación que contiene trabajos centrados en el periodo de independencia referidos principalmente a Costa Rica, Guatemala, Nicaragua y México, en los que sus autores prosiguen la escritura de una historia politica de la independencia y la formación de estados nacionales en Centroamérica vistos desde diversas dimensiones: el poder político y las identidades ciudadanas, los estados y las instituciones, la cultura y la sociedad, el periodo de la república federal y un trabajo sobre el proceso de independencia en México. ${ }^{89}$ El historiador costarricense Adrián Chaves Marín y la historiadora hondureña Elizet Payne Iglesias, coordinadores de la publicación, consideran que su aparición dará a conocer la consolidación de las nuevas ópticas que hay dentro de la historiografia centroamericana sobre la independencia y el diálogo que mantienen algunas instituciones culturales con universidades centroamericanas para divulgar a públicos más amplios los conocimientos existentes sobre el pasado regional, especialmente en los inicios de su Historia republicana. Otra contribución especializada al estudio de la independencia en la región es el libro publicado por el Instituto de Investigaciones Históricas, Antropológicas y Arqueológicas (IIHAA) de la Universidad de San Carlos de Guatemala bajo dirección del historiador guatemalteco Danilo Dardón. Por los caminos de la nación, publicado en 2020,90 pretende contribuir a la reflexión sobre la Historia de Guatemala de cara al bicentenario e incluye contribuciones dedicadas al estudio de los cambios politicos, las relaciones sociales, las imágenes y la enseñanza de la geografia dentro del periodo. También se incluye un estudio sobre la presencia de la independencia en el cine centroamericano y latinoamericano y otros artículos dedicados a temáticas diversas con los que se

87 Jordana Dym y Sajid Alfredo Herrera, op. cit.

88 Dos estudios de interés sobre opinión pública producidos durante el periodo de revisión son: Jorge Chen Sham, "En los albores de la independencia: opinión pública y sátira politica en E1 Genio de la Libertad (Lunes 10 de septiembre de 1821)", Revista Iberoamericana, n 262, 2018 , pp. 81-91. Sajid Herrera, "Espacios y opinión pública durante la anexión del Reino de Guatemala a México: San Salvador, 1821", Liminar, n 1, 2019, pp. 15-32.

89 Adrián Chaves Marín y Elizet Payne Iglesias, Reflexiones en torno al bicentenario de las independencias centroamericanas. Independencias y formación de los Estados Nacionales, 1821 1860, Museo Histórico Cultural Juan Santamaria, Alajuela, 2018.

90 Danilo Dardón (ed.), Por los caminos de la Nación, 1. Aportes a la docencia. Colección Bicentenario, Instituto de Investigaciones Históricas, Antropológicas y Arqueológicas (IIHAA) Universidad de San Carlos de Guatemala (USAC), Guatemala, 2020. 
quiere mostrar la diversidad de perspectivas desde la que se puede abordar el proceso de definición nacional guatemalteca y centroamericana.

La escritura histórica sobre la independencia en la región sigue en movimiento. Para este año se contempla dentro de la colección de la editorial Sílex dedicada a las independencias en América la publicación de un tomo específico sobre Centroamérica coordinado por Sajid Herrera y Elizet Payne en el que también tendrán cabida trabajos sobre Panamá y Chiapas, los que serán escritos por María Eugenia Claps Arenas, Armando Méndez Zárate, Sajid Herrera Mena, Leonel Hernández Sánchez, Ethel García Buchard, Xiomara Avendaño Rojas, Guillermo Fernández Ampié, Elizet Payne Iglesias y Félix Chirú Barrios. ${ }^{91}$ La Universidad Rafael Landivar planea la publicación de tres números monográficos especiales de su revista Eutopía ${ }^{92}$ dedicados al bicentenario: el primero dedicado al análisis del proceso de independencia, el segundo dedicado a los protagonismos y ausencias de los diversos grupos sociales y un tercer volumen dirigido a una reflexión contemporánea sobre el bicentenario, dimensión que ha estado bastante ausente en las muy numerosas iniciativas y actividades académicas que se han organizado recientemente. ${ }^{93}$

Este largo recorrido atestigua la presencia actual de una historiografia que ha conseguido construir un modelo explicativo complejo de la independencia de Centroamérica, en el que se propone una narración más rica y multidimensional del proceso bajo una mirada continental y horizontes de tiempo diversos para comprender las continuidades y rupturas de los procesos de cambio hacia prácticas y culturas politicas modernas. Las nacientes sociedades centroamericanas abrazaban un primer liberalismo que se trasvasaba con las herencias del antiguo régimen en el que los regionalismos y nacionalismos contribuyeron a su desintegración. La escritura histórica sobre la independencia de Centroamérica es sin lugar a dudas más profunda y diversa en sus métodos y acercamientos, en la que se han superado las visiones de la independencia pacífica, se han construido narrativas históricas extraordinariamente detalladas sobre los conflictos politicos en gran parte del istmo, se ha visibilizado a los sectores sociales subalternos para abandonar los enfoques centrados en las élites, se ha cuestionado la idea de la independencia forzada desde el exterior al comprender la dimensión internacional que cobró la expansión del proyecto imperial mexicano y se tiene una comprensión densa de los procesos de construcción de institucionalidad y la evolución en el tiempo de los conceptos de ciudadanía y representación política. Se asiente con lo vertido por Aaron Pollack

91 Sajid Herrera y Elizet Payne (coords.), La justa libertad de los pueblos. Las independencias en Centroamérica, Chiapas y Panamá, Sílex Ediciones, Madrid, 2021 (en prensa).

92 Belinda Ramos, Leticia González y Luis Pedro Taracena, Revista Eutopía. El hecho/proceso de independencia. Creación y recreación de la Historia, $\mathrm{n}^{\circ}$ 1, 2021 (en prensa). Belinda Ramos, Leticia González y Luis Pedro Taracena, Revista Eutopía. El proceso de independencia. Protagonismos y ausencias, $\mathrm{n}^{\circ}$ 2, 2021 (en prensa). Belinda Ramos, Leticia González y Luis Pedro Taracena, Revista Eutopía. 200 años después. Mirando hacia el futuro, n 3, 2021 (en prensa).

93 Según registros personales del historiador hondureño Ismael Zepeda, sólo en el 2020 se organizaron más de sesenta actividades académicas en torno al bicentenario de la independencia de Centroamérica, en las que se puede apreciar una atención mayoritaria a reflexionar sobre los hechos políticos del siglo XIX en la región dejando de lado su proyección económica, política, social y cultural actual. Ismael Zepeda, Congresos, conferencias y conversatorios sobre el Bicentenario de Centroamérica, 1821-2021, Año 2020 (documentación personal). 
al valorar que las independencias hispanoamericanas constituyen un campo historiográfico dificil en el que la escritura histórica centroamericana ha ejercido un importante potencial explicativo centrado en su dimensión social en la que los criollos ya no aparecen como únicos motores de los procesos autonomistas e independentistas, sino que ahora se perciben los papeles, a veces protagónicos, de sectores populares afrodescendientes, mestizos e indígenas durante las primeras décadas del siglo XIX. Finalmente, la presencia de una Nueva Historia Política como corriente dinamizadora de la historiografia centroamericana no tiene ningún sentido sin su integración a otras corrientes y campos historiográficos para un diálogo que produzca una nueva sintesis entre las primeras generaciones de historiadores que enfatizaban lo social y económico dentro de la larga duración y las nuevas, que se enfocan en un análisis preciso del periodo referido a las instituciones y los lenguajes políticos, proceso que se puede constituir en un intento por revisitar y comprender más profundamente la totalidad de la evolución histórica centroamericana en el siglo XIX y en la actualidad. ${ }^{94}$

Enviado el (Submission Date): 01/05/2021

Aceptado el (Acceptance Date): 8/05/2021

94 Aaron Pollack (coord.), La época de las independencias en Centroamérica y Chiapas. Procesos políticos y sociales, Instituto Mora - Universidad Autónoma Metropolitana (UAM), México, 2013, pp. 50-52. 